\title{
Fast and Versatile Inverse Hysteresis Model in Quasi-Static Regime: Derivation and
} Implementation in Simulink and PEEC Frameworks

\author{
Astorino, Antonio; Romano, D.; Antonini, G.
}

Published in:

I E E E Transactions on Magnetics

Link to article, DOI:

10.1109/TMAG.2018.2822624

Publication date:

2018

Document Version

Peer reviewed version

Link back to DTU Orbit

Citation (APA):

Astorino, A., Romano, D., \& Antonini, G. (2018). Fast and Versatile Inverse Hysteresis Model in Quasi-Static Regime: Derivation and Implementation in Simulink and PEEC Frameworks. I E E E Transactions on Magnetics, 54(7). https://doi.org/10.1109/TMAG.2018.2822624

\section{General rights}

Copyright and moral rights for the publications made accessible in the public portal are retained by the authors and/or other copyright owners and it is a condition of accessing publications that users recognise and abide by the legal requirements associated with these rights.

- Users may download and print one copy of any publication from the public portal for the purpose of private study or research.

- You may not further distribute the material or use it for any profit-making activity or commercial gain

- You may freely distribute the URL identifying the publication in the public portal 


\title{
Fast and Versatile Inverse Hysteresis Model in Quasi-Static Regime: Derivation and Implementation in Simulink and PEEC Frameworks
}

\author{
A. Astorino ${ }^{(1}$, D. Romano ${ }^{2}$, and G. Antonini ${ }^{(12}$ \\ ${ }^{1}$ DTU Fotonik, Technical University of Denmark, 2800 Kongens Lyngby, Denmark \\ ${ }^{2}$ Dipartimento di Ingegneria Industriale e dell'Informazione e di Economia, Università degli Studi dell'Aquila, \\ 67100 L'Aquila, Italy
}

\begin{abstract}
In this paper, a fast $H(M)$ magnetostatic hysteresis model is presented, tested, and used in Simulink and partial element equivalent circuit (PEEC) environments. The model is primarily designed to achieve full compatibility with the current 3-D PEEC formalism for nonlinear isotropic magnetic materials, compatibility not met by any direct model. The capabilities of the proposed model are shown in stand-alone configuration and as a core of circuit components within the Simulink environment. Its effectiveness in the 3-D PEEC formulation is then demonstrated.
\end{abstract}

Index Terms-Magnetic fields, magnetic hysteresis, magnetic material modeling, partial element equivalent circuit (PEEC) method, Simulink Simscape power systems.

\section{INTRODUCTION}

$\mathbf{T}$ HE hysteretic constitutive relation between magnetization and magnetic field in magnetic materials remains one of the hardest problems in electromagnetic modeling. Over the years, many different methods have been proposed and a vast study exists about this topic (see [1]-[4] and the references therein). The Preisach model [3] or its inverse version [5] provides an accurate macroscopic description for magnetic hysteresis, but its usage is limited by its very high computational complexity. The original Jiles-Atherton (J-A) model [6] and subsequent modified versions (see [7]-[9]) are widely adopted. The inverse version of the J-A model is also available [10].

More recently, alternative behavioral models of hysteresis have been proposed. Among them, the history-dependent hysteresis models are presented in [11] and [12] wherein the major loop and first-order reversal curves (FORCs) are represented by splines. This representation permits these curves to be described as accurately as desired. Higher order curves can then be obtained by copying and inserting a portion of the experimental or intuitively generated FORCs. Despite the accuracy and conceptual simplicity of the transplantation models proposed in [11] and [12], their disadvantage is the relative complexity of the implementation algorithm. A valuable extension of [11] and [12] is presented in [13] where spline approximations for the magnetization curves make these models applicable to major loops of any shape, in both historydependent and history-independent models of magnetic hysteresis. Such approaches have then been implemented in the electromagnetic transients program environment, as described in [14] and [15].

Manuscript received January 9, 2018; revised March 4, 2018 and March 14, 2018; accepted March 15, 2018. Corresponding author: A. Astorino (e-mail: antonio.astorino.ing@gmail.com).

Color versions of one or more of the figures in this paper are available online at http://ieeexplore.ieee.org.

Digital Object Identifier 10.1109/TMAG.2018.2822624
A further behavioral model of hysteresis has been presented in [16]. This model does not require any differential equation to be solved, because all $B-H$ curves are expressed by explicit functions, while the memory effect is modeled using a software memory instead of mathematical integration. The modeling technique is founded upon the macroscopic rules describing the qualitative trend of magnetic hysteresis curves [17], first published in 1905 by the German physicist Madelung [18]. A comprehensive and thorough review of Madelung's rules is presented in [19].

In order to make any hysteretic model of practical use to simulate electrical devices, the model has to be efficiently integrable in either a circuit simulator or a 3-D solver of Maxwell's equations. This is achievable if the model can reproduce any possible hysteresis curve, the underlying equations do not have singularities, and the computational complexity is sufficiently low. All these conditions have already been met by the $M(H)$ model in [16]. However, in some contexts, an inverse model $H(M)$ is required. This is the case of the 3-D partial element equivalent circuit (PEEC) method [20]. The PEEC formulation of linear magnetic materials has been presented in [21] and then extended to nonlinear isotropic magnetic materials in [22]. In order to inherit all the features of such a PEEC formulation in the case of hysteretic materials, an $H(M)$ model based on [16] has been developed. Its derivation and use in the PEEC framework are reported here for the first time.

Although the main goal of this paper is to demonstrate the capability of the proposed hysteresis model to be part of the 3-D PEEC method, the transition from stand-alone configuration to PEEC framework is reached through an intermediate step, which is necessary to evaluate the model functionality in a circuit context. In particular, the developed $H(M)$ transfer function is used to simulate the magnetic core of custom Simulink circuit components. Such a functionality test is believed to be paramount, as it ensures the model effectiveness in larger scenarios such as the PEEC framework. In fact, in the PEEC method, electromagnetic domains are converted into 
equivalent circuits, and the role of the hysteresis model is to perform, at any time step, the part of this conversion related to magnetic elementary domains.

This paper is organized as follows. The developed $H(M)$ model is introduced in Section II. Its integration in Simulink to model hysteretic inductors and transformers is reported in Section III, along with two numerical examples, a comparison with measurements and the model performance analysis. Section IV is devoted to the model implementation in the PEEC environment, whose effectiveness is proven by the reported numerical results. The conclusions are drawn in Section V.

\section{INVERSE HySTERESIS MODEL}

The magnetic hysteresis model proposed here is based on the modeling approach discussed in [16], wherein a scalar $M(H)$ model has been presented as an example. This means that the rules on which the behavior of the two models is based are identical. Therefore, also the memory effect of magnetic hysteresis is implemented in exactly the same way in both the $H(M)$ and the $M(H)$ models. Thus, the details regarding the model behavior and memory management are omitted here for brevity as they can be found in [16]. The derivation of the inverse model from that in [16] is as follows.

In the direct model, the unique formula adopted in to describe all the hysteresis curves is

$$
\begin{aligned}
M(H)= & \delta \alpha M_{\max }\left\{(1-m) \frac{2}{\pi}\right. \\
& \left.\cdot \arctan \left[\gamma \Theta(1-m)\left(\frac{\delta \cdot H}{H_{\text {ref }}}-1\right)\right]+m\right\}+\beta
\end{aligned}
$$

where $M_{\max }, H_{\text {ref }}$, and $\Theta$, all $>0$, are model input parameters, whereas $\alpha, \beta, \gamma, m$, and $\delta$ are variables computed by the algorithm. In particular, the value of $\delta$ is set equal to +1 if any ascending curve is produced and -1 in case of descending curves.

Here, (1) is used to calculate some parameters (as discussed later on) and find the main function of the inverse model $H(M)$. Such a function is simply obtained by solving (1) for $H$, yielding

$$
\begin{aligned}
H(M)=\delta H_{\text {ref }} & \left\{\frac{1}{\gamma \Theta(1-m)}\right. \\
\cdot & \left.\tan \left[\frac{\pi}{2(1-m)}\left(\frac{\delta(M-\beta)}{\alpha M_{\max }}-m\right)\right]+1\right\} .
\end{aligned}
$$

The domain of (2) is the open interval $\left(-M_{\max }, M_{\max }\right)$. In complete analogy with the direct model, this function generates all possible hysteresis paths, classified, according to [16], as follows.

1) Ascending or Descending Initial Magnetization Curve (AIMC or DIMC): Ascending or descending curve, respectively, starting from a thermally demagnetized state.

2) Inner Loop Curve (ILC): A curve obtained by reversing the AIMC or the DIMC.

3) nth Inversion Curve (nthIC): Any curve starting from an $(n-1)$ thIC, where $n>2$ is an integer number; the parent curve of a first inversion curve is instead an inner loop curve (ILC).

In this section, the generation of the listed curves by means of (2) is detailed.

\section{A. Initial Magnetization Curves}

The AIMC and DIMC are obtained by substituting in (2) $\alpha=1, \beta=0, \gamma=1$, and $m=m_{0}$, yielding

$$
\begin{aligned}
H(M)=\delta H_{\text {ref }}\left\{\frac{1}{\Theta\left(1-m_{0}\right)}\right. \\
\left.\cdot \tan \left[\frac{\pi}{2\left(1-m_{0}\right)}\left(\frac{\delta M}{M_{\max }}-m_{0}\right)\right]+1\right\}
\end{aligned}
$$

where $m_{0}$ is a constant such that (3) passes through the origin of the reference frame, independently of the sign of $\delta$. The value of $m_{0}$ is numerically calculated only once.

The initial curves generated by the $H(M)$ model are exactly the same as those obtained with the $M(H)$ model. However, the AIMC and DIMC are the only cases in which the two models coincide.

\section{B. Inner Loop Curves}

Let $P_{\text {tip }}=\left(H_{\text {tip }}, M_{\text {tip }}\right)$ be a reversal point on either the AIMC or the DIMC. The new curve is, by definition, an ILC. Following the approach proposed in [16], the ILC born to $P_{\text {tip }}$ is generated by using (2), setting $\alpha=1, \beta=0$, and $\gamma=1$, and changing the sign of $\delta$. The parameter $m$, instead, becomes a function of $M$, whose image varies from an initial value, denoted by $m_{\text {tip }}$, to $m_{0}$, while $M$ is moving from $M_{\text {tip }}$ to $-M_{\text {tip }}$. The function adopted here is

$$
m(M)=\left(m_{0}-m_{\text {tip }}\right) \cdot\left[\frac{1}{2} \cos \left(\pi \frac{\delta M-\left|M_{\text {tip }}\right|}{2\left|M_{\text {tip }}\right|}\right)+\frac{1}{2}\right]^{w_{1}}+m_{\text {tip }}
$$

where the parameter $w_{1}$ is to be determined by fitting some measured ILCs or intuitively. For any ILC, (2) becomes

$$
\begin{aligned}
H(M)= & \delta H_{\text {ref }}\left\{\frac{1}{\Theta(1-m(M))}\right. \\
& \left.\cdot \tan \left[\frac{\pi}{2(1-m(M))}\left(\frac{\delta \cdot M}{M_{\max }}-m(M)\right)\right]+1\right\}
\end{aligned}
$$

where $m_{\text {tip }}$ is numerically computed imposing $H\left(M_{\text {tip }}\right)=$ $H_{\text {tip }}$ in (5). This calculation is required only when an initial magnetization curve is reversed.

Note that (5) is an implicit function of $M$ and the corresponding equation in the direct model is an implicit function of $H$. This is the obvious reason why the two functions cannot be analytically derived from one another. However, this does not prevent the general modeling approach proposed in [16] from being applied to the inverse model presented here.

By definition, closed loops generated by pairs of ILCs are called ILs. As an example, the AIMC and some ILs are depicted in Fig. 1 for a given choice of model parameters. 


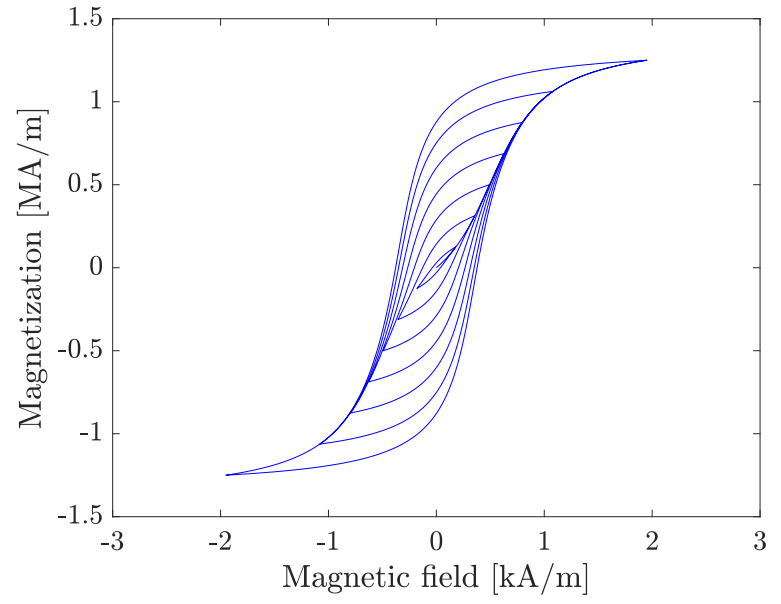

Fig. 1. Example of AIMC and ILs. The model parameters are $M_{\max }=$ $1.4 \times 10^{6} \mathrm{Am}^{-1}, H_{\text {ref }}=500 \mathrm{Am}^{-1}, \Theta=2$, and $w_{1}=1$.

\section{Inversion Curves}

By definition, an $n$ thIC exists if and only if an ILC has been already generated. In the proposed model, the values of $m_{1}$ and $M_{\text {tip }}$ regarding the last-produced ILC are used to determine the values of $\alpha, \beta$, and $\gamma$ to substitute in (2) in order to generate the $n$ thIC of interest. To this end, let $P^{(i)}=\left(H^{(i)}, M^{(i)}\right)$ be the starting point of such a curve and $P^{(f)}=\left(H^{(f)}, M^{(f)}\right)$ be the starting point of the previous curve [an $(n-1)$ thIC or an ILC]. According to the memory rules, the considered $n$ thIC tends to $P^{(f)}$. Let also $\gamma_{i, f}$ be given by

$$
\gamma_{i, f}=\left(\frac{M^{(f)}-M^{(i)}}{M^{(f)}+\delta\left|M_{\mathrm{tip}}\right|}\right)^{w_{2}}
$$

where $w_{2}$ is a shape parameter whose evaluation is described in [16]. Substituting in (1) $m=m\left(M^{(i)}\right), \alpha=1, \beta=0$, and $\gamma=\gamma_{i, f}$, the auxiliary parameter $\tilde{M}^{(i)}=M\left(H^{(i)}\right)$ is calculated. Similarly, substituting in (1) $m=m\left(M^{(f)}\right)$ and leaving the other parameters unchanged, $\tilde{M}^{(f)}=M\left(H^{(f)}\right)$ is obtained. Let then $\alpha_{i, f}$ and $\beta_{i, f}$ be given by

$$
\begin{aligned}
& \alpha_{i, f}=\frac{M^{(f)}-M^{(i)}}{\tilde{M}^{(f)}-\tilde{M}^{(i)}} \\
& \beta_{i, f}=M^{(i)}-\alpha_{i, f} \tilde{M}^{(i)} .
\end{aligned}
$$

Substituting (4), (6a), (6b), and (6c) for $m, \gamma, \alpha$, and $\beta$, respectively, in (2), the $n$ thIC connecting $P^{(i)}$ to $P^{(f)}$ is

$$
\begin{aligned}
& H(M)=\delta H_{\operatorname{ref}}\left\{\frac{1}{\gamma_{i, f} \Theta(1-m(M))}\right. \\
&\left.\cdot \tan \left[\frac{\pi}{2(1-m(M))}\left(\frac{\delta\left(M-\beta_{i, f}\right)}{\alpha_{i, f} M_{\max }}-m(M)\right)\right]+1\right\} .
\end{aligned}
$$

Loops composed of at least one inversion curve are called minor loops (MLs). Some examples of hysteresis curves produced by the proposed model are depicted in Fig. 2, showing, in particular, families of the second inversion curves (2ndICs) and third inversion curves ( 3 rdICs), and the MLs involving them.

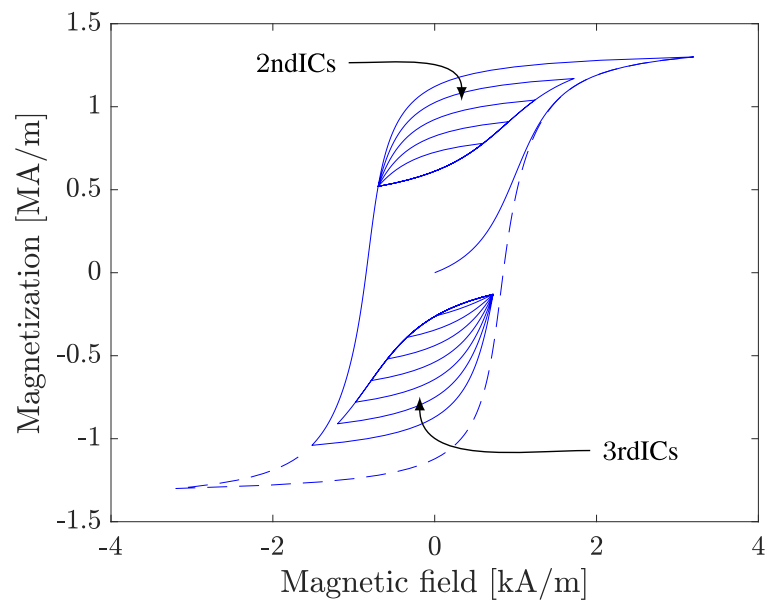

Fig. 2. Example of families of the 2ndICs and 3rdICs. The model parameters are $M_{\max }=1.4 \times 10^{6} \mathrm{Am}^{-1}, H_{\mathrm{ref}}=1 \times 10^{3} \mathrm{Am}^{-1}, \Theta=4, w_{1}=1$, and $w_{2}=0.45$. The path starts from the origin, from which the AIMC is produced. Then, a family of 2ndICs and a family of 3rdICs are generated. The dashed line is added to show the IL within which all the generated curves are located.

\section{Model IMPLEMENTATION IN SimUlink AND PERFORMANCE ANALYSIS}

In this section, a robustness test performed on the developed model in the stand-alone configuration is presented. Such a test is preliminary to the implementation of the model as a magnetic core of inductors or two-winding transformers in Simulink. The steps toward this implementation are also illustrated in this section. Finally, the speed of the resulting Simulink models is evaluated.

Although within the Simulink environment, there are several libraries for circuit simulations, here selected. However, the process that leads from a Simulink block to a circuit component is, with minor variations, applicable to any other library.

\section{A. Robustness Test}

In general, when an iterative solver is looking for the system solution at a given time step, many attempts may be needed. At each attempt, all the functions in the system have to give a consistent response, even when the initial guess is far from being the correct one. This is particularly challenging in case of hysteresis models, as they do not represent injective functions and the memory effect that rules their behavior may fail when subject to exceptional inputs, such as those normally generated by iterative solvers.

In order to demonstrate the capability of the developed model to cope with any sort of input signal, the model in the stand-alone configuration is fed with a sequence of 16000 random values with uniform distribution, ranging within the model input domain. The test is run 20 times, and the average wall-clock time is calculated to be about $0.58 \mathrm{~s}, 36 \mu$ s per time step on a machine with a $2.9 \mathrm{GHz}$ Intel Core i7 processor. Beside the absence of bugs, the consistency of all calculated points is demonstrated by the resulting $M-H$ plots, as the example reported in Fig. 3. As shown, all points are located 


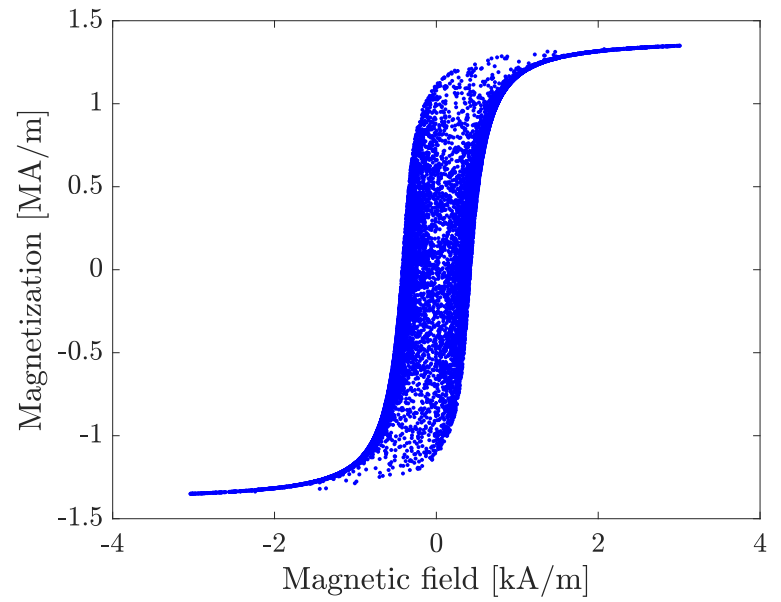

Fig. 3. $M-H$ graph obtained by using the developed hysteresis model with parameters $M_{\max }=1.4 \times 10^{6} \mathrm{Am}^{-1}, H_{\text {ref }}=500 \mathrm{Am}^{-1}, \Theta=3.5, w_{1}=1$, and $w_{2}=0.45$ and random input with uniform distribution ranging in the interval $\left(-1.35 \times 10^{6}, 1.35 \times 10^{6}\right) \mathrm{Am}^{-1}$.

inside a defined IL, whose tips are determined by the extrema of the chosen range for the random sequence.

\section{B. Simulink Hysteretic Inductor Design}

1) Equations: A hysteretic cylindrical inductor with a core cross section $S$, core length $\ell$, and number of turns $N_{\mathrm{t}}$ is considered. The voltage drop across the inductor is denoted by $v_{\mathrm{L}}$, whereas the current flowing through its winding is indicated by $i_{\mathrm{L}}$. Neglecting any parasitic phenomenon, such as leakage flux and eddy currents, the following relations hold:

$$
\left\{\begin{array}{l}
\frac{B(t)}{\mu_{0}}=\frac{1}{\mu_{0} S N_{\mathrm{t}}} \int_{0}^{t} v_{\mathrm{L}}\left(t^{\prime}\right) d t^{\prime} \\
i_{\mathrm{L}}(t)=H(t) \frac{\ell}{N_{\mathrm{t}}}
\end{array}\right.
$$

where $H$ is calculated by the hysteresis model, $\mu_{0}$ is the vacuum magnetic permeability, and $B$ is the magnetic flux density satisfying

$$
\frac{B(t)}{\mu_{0}}=H(M(t))+M(t) .
$$

2) From Equations to Block Diagram: The diagram corresponding to (8) is displayed in Fig. 4.

Such a schematic represents the inductor mathematical model implemented in Simulink. Mirroring the arrangement of terms in (8), the input of the diagram is $v_{\mathrm{L}}$. Consequently, the schematic output is $i_{\mathrm{L}}$, which is proportional to $H$. However, due to the nonlinear relation between $H$ and $M$, it is not possible to explicitly solve (9) for $H$. For this reason, the value of $H$ is used as a feedback signal to calculate itself through the hysteresis model, as shown in Fig. 4, hence creating an algebraic loop. To solve the loop, a unit delay (not shown in the diagram for simplicity) within the feedback loop is adopted.

3) From Block Diagram to Circuit Component: Due to the choice of input and output, the diagram in Fig. 4 is translated into an inductor that behaves as a current generator controlled by the voltage across itself. This is shown in Fig. 5, where the hysteretic inductor model used in this paper is depicted.

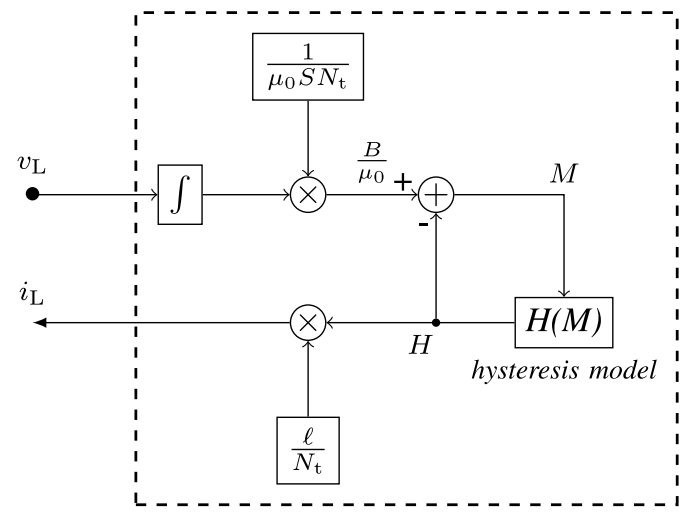

Fig. 4. Graphical representation of (8).

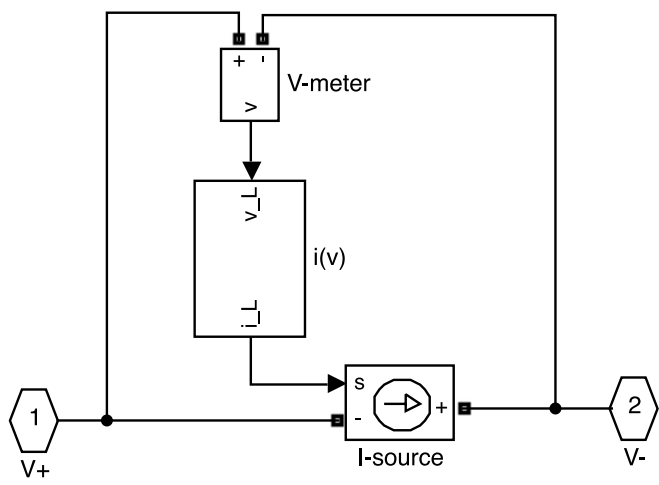

Fig. 5. Implementation of the hysteretic inductor as a voltage-controlled current generator using Simscape Power Systems in Simulink. The block labeled "i(v)" encloses the diagram in Fig. 4, "V-meter" is a voltage meter, and "I-source" is an ideal current generator, driven by the output of "i(v)."

Notice that the proposed inductor model is not suitable for open-circuit conditions, as the current source involved is ideal. However, this issue is easily solved in two ways: by using a nonideal switch (with high but not infinite resistance when open) or by placing an arbitrarily high resistor in parallel to the current source.

It is also important to observe that, when the schematic in Fig. 5 is part of a circuit, at each time step, the current depends on the voltage, which is not known yet. As a result, the solver must compute both quantities iteratively, hence reducing the overall system performance. To overcome this issue, a unit delay is placed at the input of the block "i(v)."

\section{Simulink Hysteretic Two-Winding Transformer Design}

1) Equations: A two-winding transformer with lossless coils is considered. The number of turns is denoted by $N_{\mathrm{t}}$, the voltage is denoted by $v$, and the current is denoted by $i$. The subscript 1 or 2 is added to these quantities to specify the coil they refer to, primary or secondary, respectively. The magnetic circuit is assumed to be a loop with a constant cross section $S$ and average length $\ell$. 


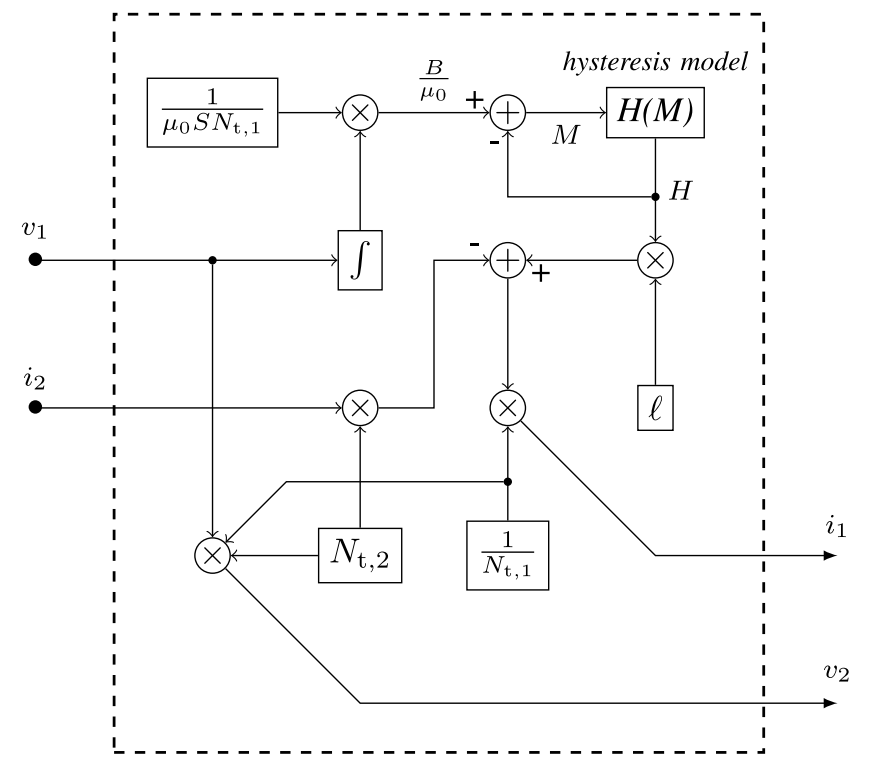

Fig. 6. Graphical representation of (10).

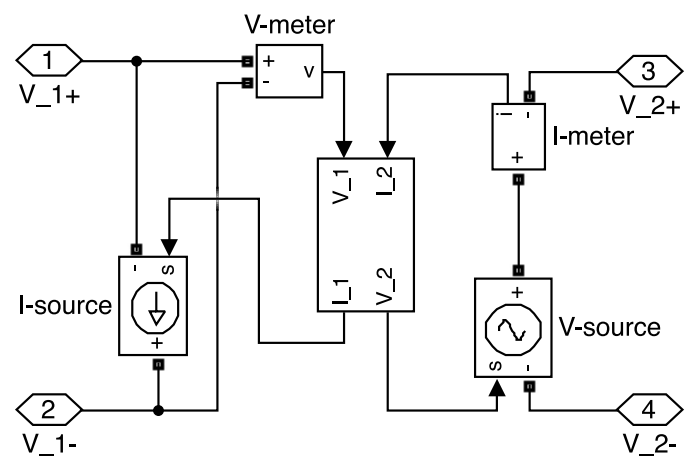

Fig. 7. Implementation of the designed hysteretic transformer as a hybrid voltage and current generator using Simscape Power Systems in Simulink. The central block encloses the diagram in Fig. 6. Current and voltage generators are denoted by "I-source" (generating $i_{1}$ ) and "V-source" (generating $v_{2}$ ), respectively. The input voltage $v_{1}$ is measured by the block "V-meter," whereas the input current $i_{2}$ is probed by the block "I-meter."

The equations of interest are

$$
\left\{\begin{array}{l}
\frac{B(t)}{\mu_{0}}=\frac{1}{\mu_{0} S N_{\mathrm{t}, 1}} \int_{0}^{t} v_{1}\left(t^{\prime}\right) d t^{\prime} \\
i_{1}(t)=\frac{\ell H(t)-N_{\mathrm{t}, 2} i_{2}(t)}{N_{\mathrm{t}, 1}} \\
v_{2}(t)=\frac{N_{\mathrm{t}, 2}}{N_{\mathrm{t}, 1}} v_{1}(t) .
\end{array}\right.
$$

2) From Equations to Block Diagram: A schematized version of (10) is depicted in Fig. 6.

The relations in (10) are arranged, such that $v_{1}$ and $i_{2}$ are inputs of the system, whereas $i_{1}$ and $v_{2}$ are outputs. The equations find a unique solution once a load is connected to the secondary coil, hence providing $i_{2}$ as a function of the generated $v_{2}$.

3) From Block Diagram to Circuit Component: As before, inputs are converted into meters and outputs into controlled generators. The result is depicted in Fig. 7.

Note that such a schematic is not symmetric. From the user point of view, the consequence is that the primary winding has to be fed by an active circuit, whereas the load of secondary

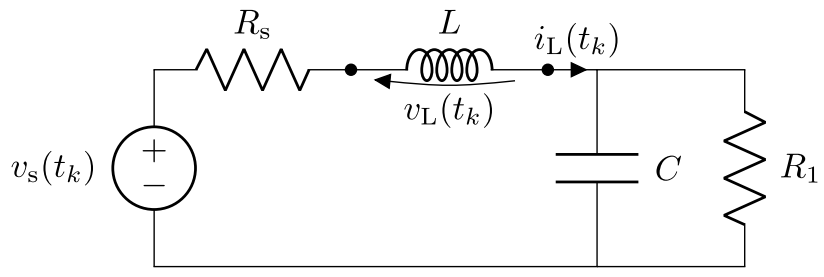

Fig. 8. Simulation III-D1: $R L C$ circuit with hysteretic inductor.

coil can be any either passive or active. Obviously, this does not entail any loss of generality as primary and secondary coils can be always defined depending on which port the active source is connected to. Also in this case, the generators in use are ideal. Therefore, to allow the open-circuit condition at the primary winding, it is necessary to place a resistor with very high resistance in parallel to the current generator. Similarly, to allow a short-circuit condition at the secondary winding, a resistor with very small resistance must be placed in series to the voltage source.

\section{Numerical Examples}

The proposed hysteresis model, used as an inductor and transformer magnetic core, is tested through a set of simulations, aiming at demonstrating the model capabilities and performance. The sampling time $\Delta_{\mathrm{t}}$ is $20 \mu \mathrm{s}$ in all tests. The continuous time variable $t$ is replaced by $t_{\mathrm{k}}=k \Delta_{\mathrm{t}}$, where $k$ runs from 0 to the number of simulation time steps, denoted by $N_{\mathrm{s}}$.

1) RLC Circuit: The schematic of the circuit analyzed in this test is depicted in Fig. 8, wherein the inductor is the custom circuit depicted in Fig. 5. The parameters chosen for this test are as follows.

1) $R_{\mathrm{S}}=50 \Omega$.

2) $R_{1}=1 \mathrm{k} \Omega$.

3) $C=1 \mathrm{mF}$.

4) $N_{\mathrm{t}}=25$.

5) $S=100 \mathrm{~mm}^{2}$.

6) $\ell=10 \mathrm{~mm}$.

7) $M_{\max }=840 \times 10^{3} \mathrm{Am}^{-1}$.

8) $H_{\mathrm{ref}}=800 \mathrm{Am}^{-1}$.

9) $\Theta=3$.

10) $w_{1}=2$.

11) $w_{2}=0.45$.

The voltage source provides the following waveform:

$$
v_{\mathrm{s}}\left(t_{\mathrm{k}}\right)=40\left|\sin \left(2 \pi f t_{\mathrm{k}}\right)\right| \quad[\mathrm{V}]
$$

where $f=50 \mathrm{~Hz}$. The simulation time is $0.24 \mathrm{~s}$, corresponding to $N_{\mathrm{S}}=12000$. The simulated inductor voltage drop and current, namely, $v_{\mathrm{L}}$ and $i_{\mathrm{L}}$, respectively, are depicted in Fig. 9(a), whereas the $B-H$ graph related to the inductor core is shown in Fig. 9(b).

2) Transformer Circuit: To test the developed hysteresis model as a transformer core, the schematic in Fig. 10 is simulated.

The parameters chosen for this simulation are as follows.

1) $R_{\mathrm{S}}=R_{1}=50 \Omega$. 

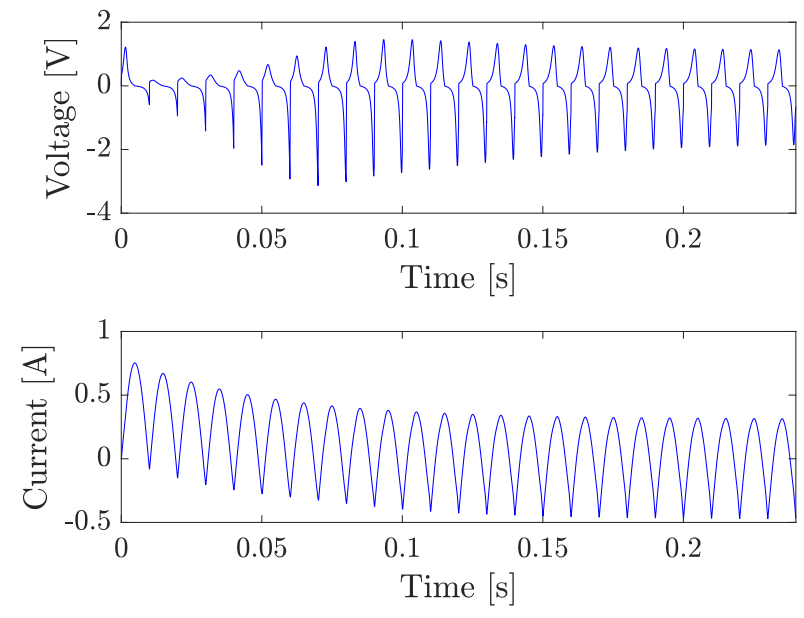

(a)

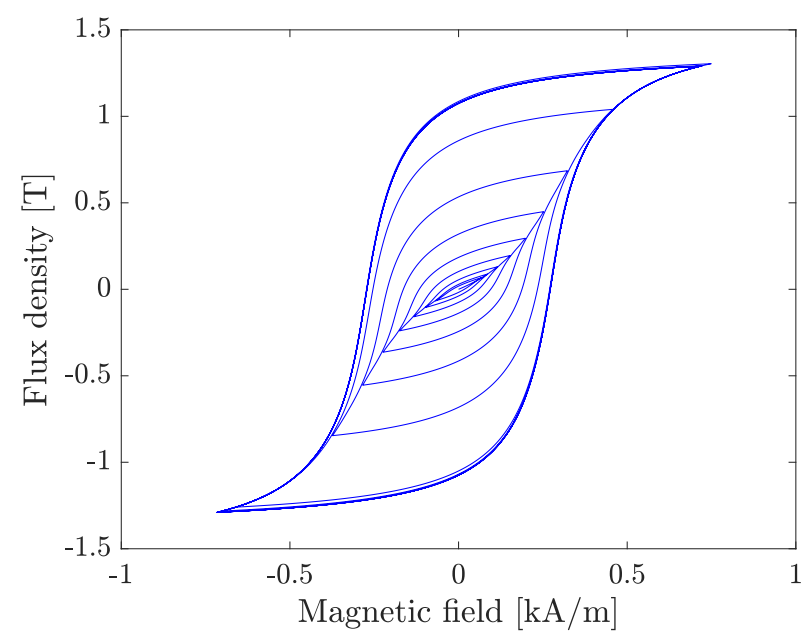

(b)

Fig. 9. Simulation III-D1. (a) Time evolution of inductor current and voltage. (b) $B-H$ curves simulated inside the inductor core, modeled using the developed hysteresis model.

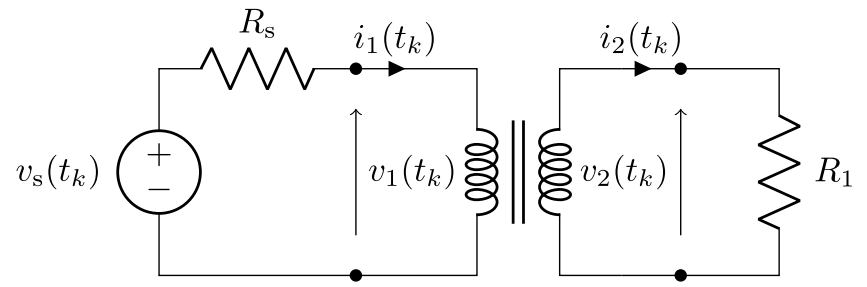

Fig. 10. Simulation III-D2: circuit for testing the developed hysteresis model as a magnetic core of a transformer.
2) $N_{\mathrm{t}, 1}=240$.
3) $N_{\mathrm{t}, 2}=80$.
4) $S=3.6 \times 10^{3} \mathrm{~m}^{2}$.
5) $\ell=0.7 \mathrm{~m}$
6) $M_{\max }=1.2 \times 10^{6} \mathrm{Am}^{-1}$.
7) $H_{\mathrm{ref}}=300 \mathrm{Am}^{-1}$.
8) $\Theta=3$.
9) $w_{1}=1$.
10) $w_{2}=0.55$.
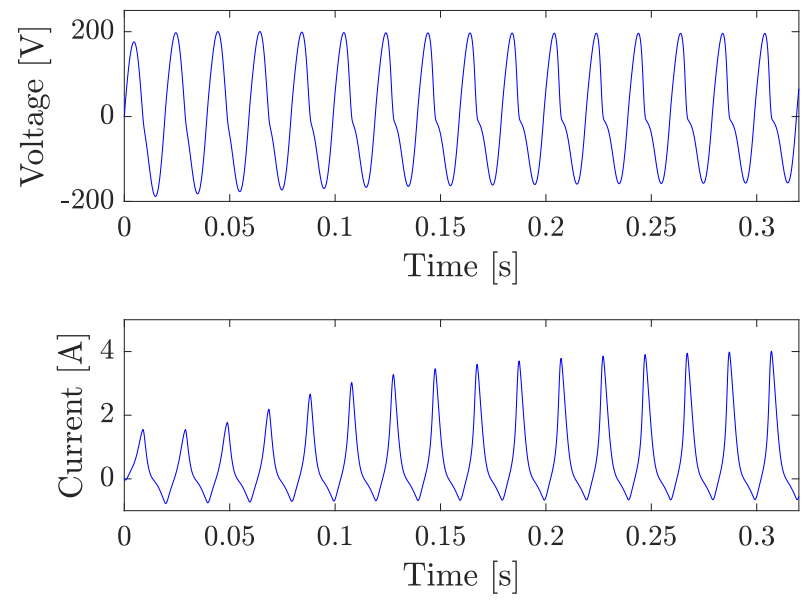

(a)

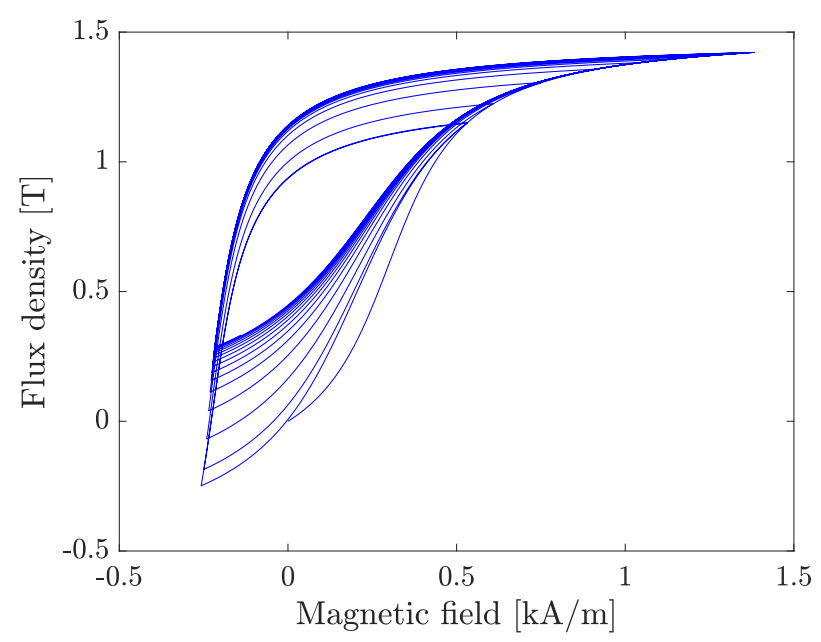

(b)

Fig. 11. Simulation III-D2. (a) Time evolution of transformer current and voltage at the primary coil. (b) $B-H$ curves simulated by the developed hysteresis model used as a transformer core.

The voltage source evolves over time according to the following equation:

$$
v_{\mathrm{s}}\left(t_{\mathrm{k}}\right)=200 \sin \left(2 \pi f t_{\mathrm{k}}\right)+40\left(1-e^{-0.2 f t_{\mathrm{k}}}\right) \quad[\mathrm{V}]
$$

which represents a sinusoid at frequency $f=50 \mathrm{~Hz}$ plus a bias that asymptotically reaches $40 \mathrm{~V}$ by slowly increasing from $0 \mathrm{~V}$.

The simulation time is $0.32 \mathrm{~s}$, corresponding to $N_{\mathrm{s}}=16000$. Simulated voltage and current at the primary winding, i.e., $v_{1}$ and $i_{1}$, are shown in Fig. 11(a). The corresponding quantities simulated at the secondary winding, namely, $v_{2}$ and $i_{2}$, are not shown for brevity, as their waveform is exactly the same as that of $v_{1}$, due to the assigned proportionality between $v_{1}$ and $v_{2}$ [see (10)] and the presence of a purely resistive load at the secondary port. The obtained $B-H$ curves are shown in Fig. 11(b).

\section{E. Comparison With Measurements}

To validate the proposed modeling approach, the $R L$ circuit in Fig. 12 is considered. 


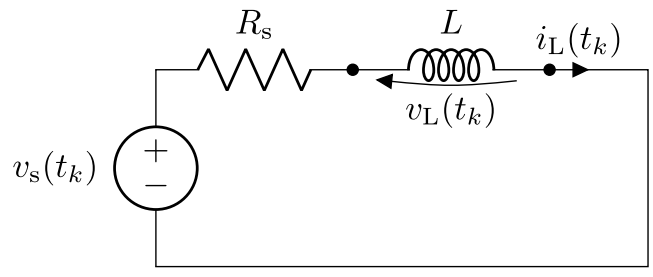

Fig. 12. Simulation III-E: $R L$ circuit with hysteretic inductor.

The device under test is the primary winding of a transformer used as an inductor (the secondary winding is left open). The resistor $R_{\mathrm{S}}$ comprises the equivalent series $(d c)$ resistance of the primary winding of $0.9 \Omega$ and the shunt resistance of $0.1 \Omega$ used to indirectly measure the current. The circuit is fed by connecting it directly to a power socket of the lab. The measured voltage is modeled as an ideal source providing the following waveform:

$$
v_{\mathrm{s}}\left(t_{\mathrm{k}}\right)=335 \sin \left(2 \pi f t_{\mathrm{k}}\right)+0.3 \quad[\mathrm{~V}]
$$

where $f=50 \mathrm{~Hz}$.

The parameters adopted in this simulation are as follows.

1) $R_{\mathrm{S}}=1 \Omega$.

2) $N_{\mathrm{t}}=229$.

3) $S=4.9 \times 10^{3} \mathrm{~m}^{2}$.

4) $\ell=0.7 \mathrm{~m}$.

5) $M_{\max }=850 \times 10^{3} \mathrm{Am}^{-1}$.

6) $H_{\text {ref }}=1500 \mathrm{Am}^{-1}$.

7) $\Theta=3$.

8) $w_{1}=1.6$.

9) $w_{2}=2$.

Apart from $v_{\mathrm{s}}, R_{\mathrm{S}}$, and $N_{\mathrm{t}}$, all the parameters have been estimated by trial and error in the attempt to achieve a good match with the measured current and calculated hysteresis loop at steady state. Therefore, such parameters must be seen as "equivalent," as they somehow summarize several parasitic phenomena (e.g., leakage flux) which could not be measured due to lack of proper equipment. The result is shown in Fig. 13.

Notice the asymmetry in both current and loops which reflect the presence of a parasitic dc component in the voltage source.

\section{F. Speed Evaluation}

Besides the complexity added by the algorithm necessary to implement the hysteresis memory effect, the system nonlinearity is an obvious cause of simulation speed reduction. Hence, in order to evaluate how the developed hysteresis model actually affects the system performance, the average elapsed real time for the circuits described in Sections III-D1 and III-D2 is calculated. The results are then compared with two simpler scenarios, namely, linear and nonlinear. In the linear case, the function $H(M)$ is replaced by

$$
H=\frac{M}{\chi}
$$

where $\chi=800$ is the (roughly) estimated average magnetic susceptibility exhibited by the core when the steady state

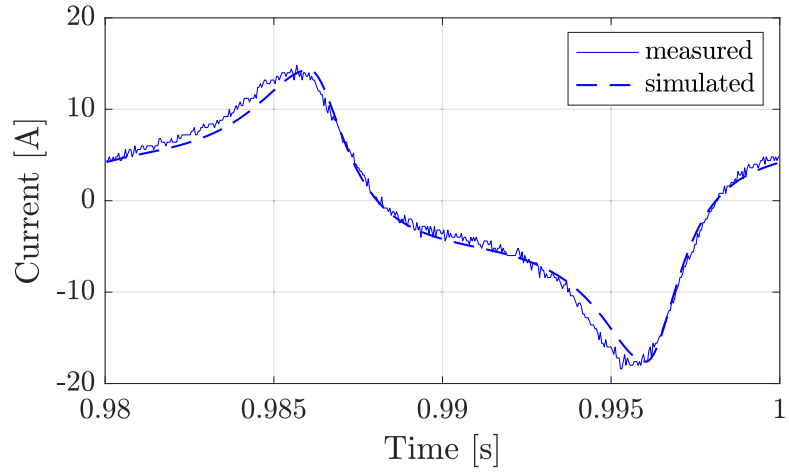

(a)

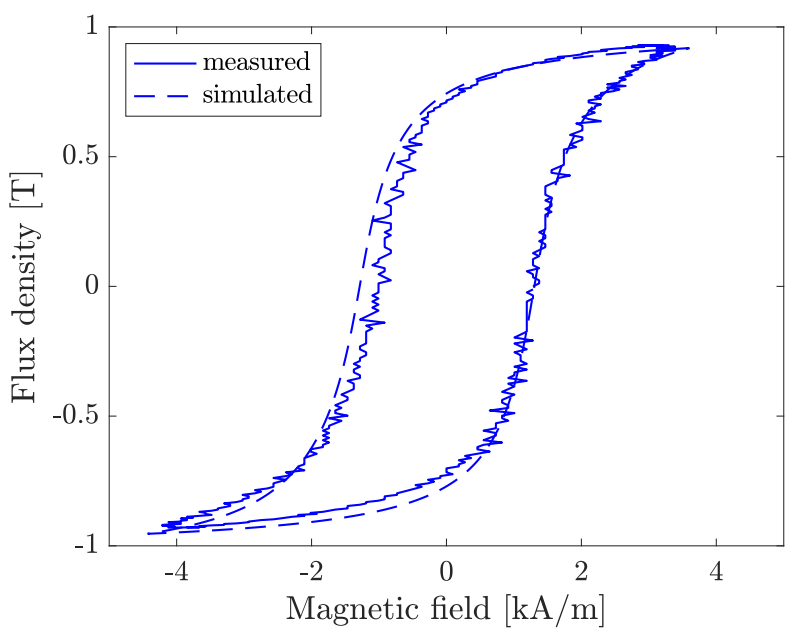

(b)

Fig. 13. Simulation III-E. (a) Time evolution of inductor current. (b) $B-H$ curves simulated inside the inductor core, modeled using the developed hysteresis model.

is reached in the hysteretic cases. In the nonlinear case, the adopted transfer function is

$$
H=\frac{M_{\max }}{\chi} \tanh ^{-1}\left(\frac{M}{M_{\max }}\right)
$$

designed to obtain a similar slope around the origin to the linear case and saturation at $M= \pm M_{\max }$.

The simulations in this section are run on a machine with a $2.9 \mathrm{GHz}$ Intel Core i7 processor. They are timed by using "tic()" and "toc()" functions placed in the Simulink "StartFcn" and "StopFcn" callbacks, respectively. The elapsed time returned by "toc()" is averaged over 20 simulations. The results are reported in Table I in terms of average total wallclock time, denoted by $T_{\text {tot }}$, and average wall-clock time per time step, indicated with $T_{\mathrm{ts}}$.

As shown, when the hysteresis model is used to characterize the magnetic core, the simulations of the $R L C$ circuit takes $35 \%$ more than the linear case. Similarly, the elapsed time increases by $25 \%$ when the hysteretic transformer is simulated compared with the linear one. Although such percentages are already acceptable, it must be observed that part of the extra time can be ascribed to the nonlinearity only, which increases the elapsed real time by about $9 \%$ in both cases. 
TABLE I

Speed Test Results

\begin{tabular}{|c|c|c|c|}
\hline \multicolumn{2}{|c|}{ Simulation } & $T_{\text {tot }}$ & $T_{\mathrm{ts}}$ \\
\hline \hline \multirow{3}{*}{ RLC } & Linear & $1.15 \mathrm{~s}$ & $97 \mu \mathrm{s}$ \\
\cline { 2 - 4 } & Nonlinear & $1.25 \mathrm{~s}$ & $104 \mu \mathrm{s}$ \\
\cline { 2 - 4 } & Hysteretic & $1.59 \mathrm{~s}$ & $132 \mu \mathrm{s}$ \\
\hline \multirow{3}{*}{ Transformer } & Linear & $1.72 \mathrm{~s}$ & $108 \mu \mathrm{s}$ \\
\cline { 2 - 4 } & Nonlinear & $1.87 \mathrm{~s}$ & $117 \mu \mathrm{s}$ \\
\cline { 2 - 4 } & Hysteretic & $2.15 \mathrm{~s}$ & $135 \mu \mathrm{s}$ \\
\hline
\end{tabular}

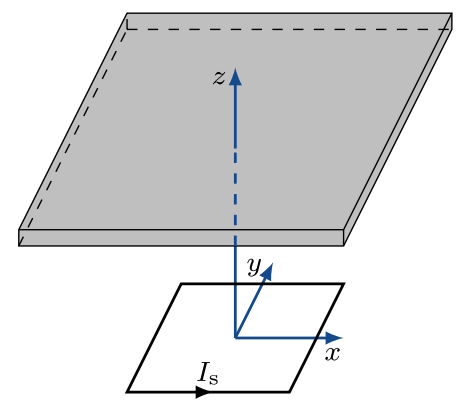

Fig. 14. Simulation IV-B1: magnetized plate excited by a square current loop (image not to scale).

\section{Model Implementation In the PEEC Framework}

Robustness, low computational complexity, and capability of being integrated in a circuit environment, demonstrated in Section III, enable the proposed model to be effectively adopted in the PEEC method. In fact, for each magnetic subdomain in the mesh, one instance of the model is generated, and the total number of subdomains grows with the desired accuracy, leading to a large number of model instances. Hence, robustness and low computational complexity are indispensable to achieve fast, or at least feasible, simulations.

The PEEC formulation adopted here is detailed in [22], wherein the use of nonlinear isotropic magnetic materials is demonstrated. In that work, the magnetization is a system unknown from which the magnetic field is calculated. Moreover, in [22], an analytical expression for the Jacobian involved in the Newton-Raphson algorithm is found, considering $M$ as one of the state variables with respect to which partial derivatives are computed. The analytical expression of the Jacobian is one of the most important achievements obtained in [22], as it enables more precise and fast simulations compared with those obtained using a numerical Jacobian. Thus, by considering an $H(M)$ model as a particular case of nonlinearity, the aforementioned features of the PEEC formulation for nonlinear magnetic materials are preserved. Nevertheless, a few modifications to the general nonlinear case are introduced. Those changes are reported in this section along with two numerical examples.

\section{A. Magnetic Permeability and Constitutive Relation}

The use of a scalar model in the 3-D PEEC framework is allowed by the PEEC formalism itself, as only one Cartesian component of the field is defined in each volume of the mesh.
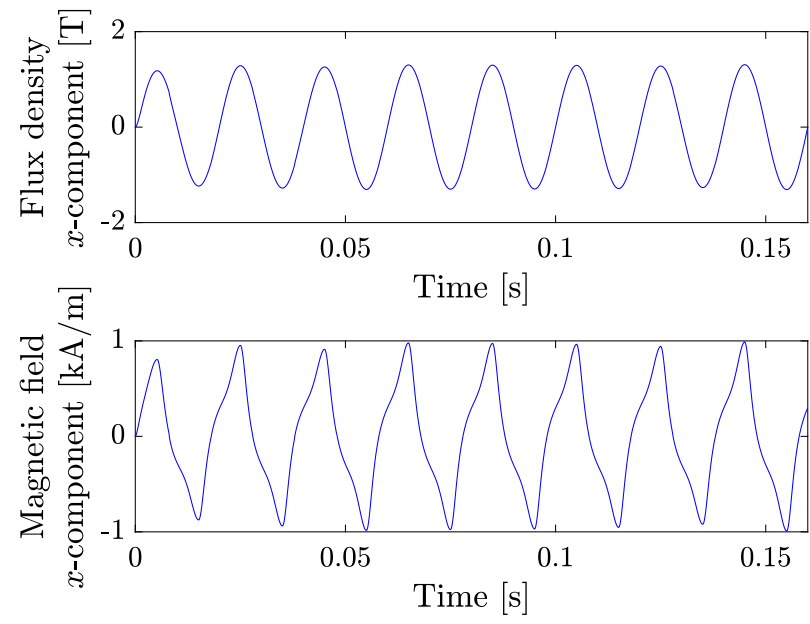

(a)

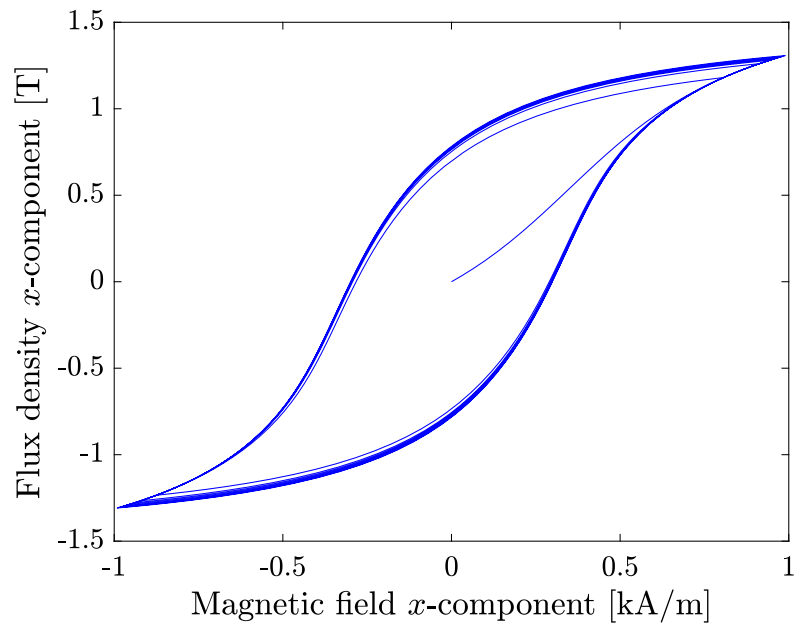

(b)

Fig. 15. Simulation IV-B1. (a) Time evolution of $B_{x}$ and $H_{x}$ at $(5,5$, $0.45)[\mathrm{mm}]$. (b) $B_{x}$ versus $H_{x}$ at the same point.

Hence, within each magnetic subdomain, the field does not rotate. In other words, $\vec{M}$ and $\vec{H}$ are treated as scalar fields, represented by their respective amplitudes, with preassigned direction. Based on these assumptions, at any point $\vec{r}$ in the $j$ th elementary magnetic volume, the (scalar) relative magnetic permeability is defined as

$$
\mu_{r, j}(t)=\frac{B_{j}(\vec{r}, t)}{H_{j}\left(M_{j}(\vec{r}, t)\right)}, \quad j=1,2, \ldots, 3 N_{m}
$$

where $B_{j}$ and $H_{j}$ are the amplitudes of the flux density $\vec{B}$ and the magnetic field $\vec{H}$ (in the $j$ th element), respectively, and $3 N_{\mathrm{m}}$ is the number of elementary magnetic volumes. Consequently, the constitutive relation characterizing magnetic materials are written as

$$
\nabla \times \vec{A}\left(\vec{r}_{j}, t\right)=\frac{\mu_{r, j}(t) \mu_{0}}{\mu_{r, j}(t)-1} \vec{M}\left(\vec{r}_{j}, t\right), \quad j=1,2, \ldots, 3 N_{m}
$$

where $\vec{r}_{j}$ denotes the subset of points in the $j$ th elementary magnetic volume. 

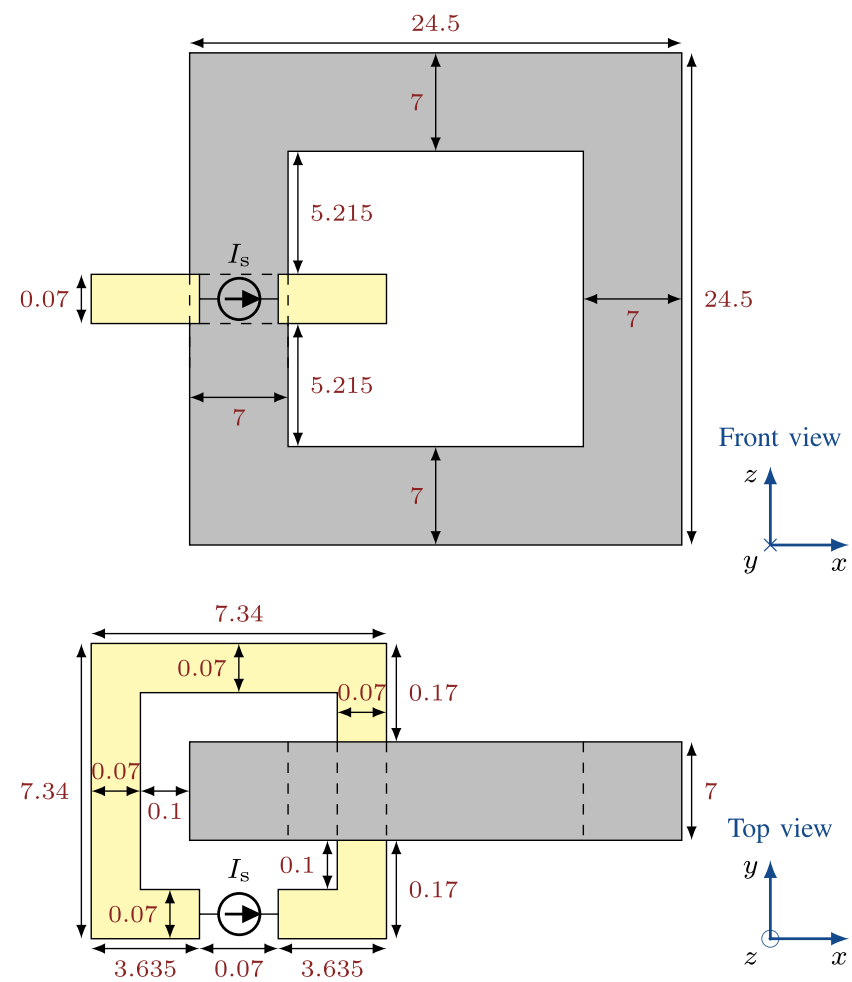

Fig. 16. Simulation IV-B2: closed magnetic circuit (not to scale). All dimensions are in $\mathrm{mm}$.

In [22], the equation corresponding to (17) has exactly the same form. However, it is necessary to highlight that, in this paper, the term $\left(\mu_{\mathrm{r}, j}-1\right)$ may equal zero, according to (16), even when the magnetic field is not zero because of the hysteretic behavior. In this case (although extremely rare when $t$ is discretized and finite-precision computation is adopted), the value of $M_{j}$ is artificially perturbed, such that the corresponding permeability is different from zero. In the nonhysteretic case, instead, this is not necessary because the magnetization is zero if and only if also the magnetic field is zero. Consequently, the curl of the vector potential, i.e., the flux density, is also zero, meaning that (17) has an immediate solution.

The perturbative technique adopted to avoid division by zero in (17) is also applied to $H_{j}$ in (16).

\section{B. Numerical Examples}

Two simulations are presented. In both, a sample of magnetic material is subject to the field produced by a coil carrying an electric current. The simulations are performed using a computer with four Intel i7-5500U-CPU2.9 GHz processors.

1) Magnetic Plate: A current loop is placed in proximity to the magnetic plate under test, as schematically represented in Fig. 14. The coil is a square, infinitesimally thin loop with a side length of $10 \mathrm{~mm}$. The loop lies on the $X Y$-plane and its center coincides with the origin of the coordinate system. The plate dimensions are $20 \mathrm{~mm}$ in the $x$ - and $y$-directions, and $0.2 \mathrm{~mm}$ in the $z$-direction. The bottom surface of the plate is $0.35 \mathrm{~mm}$ above and parallel to the $X Y$-plane, and its center is along the $z$-axis of the coordinate system.
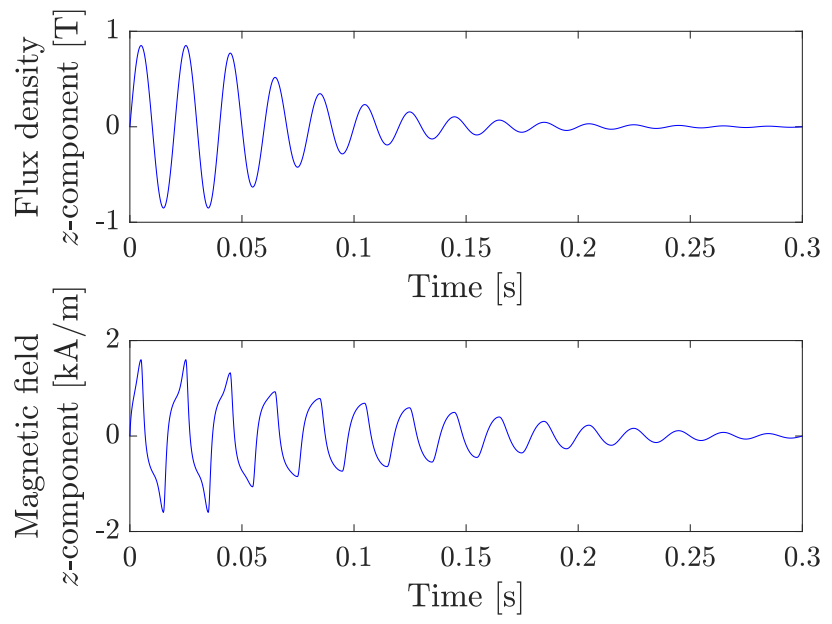

(a)

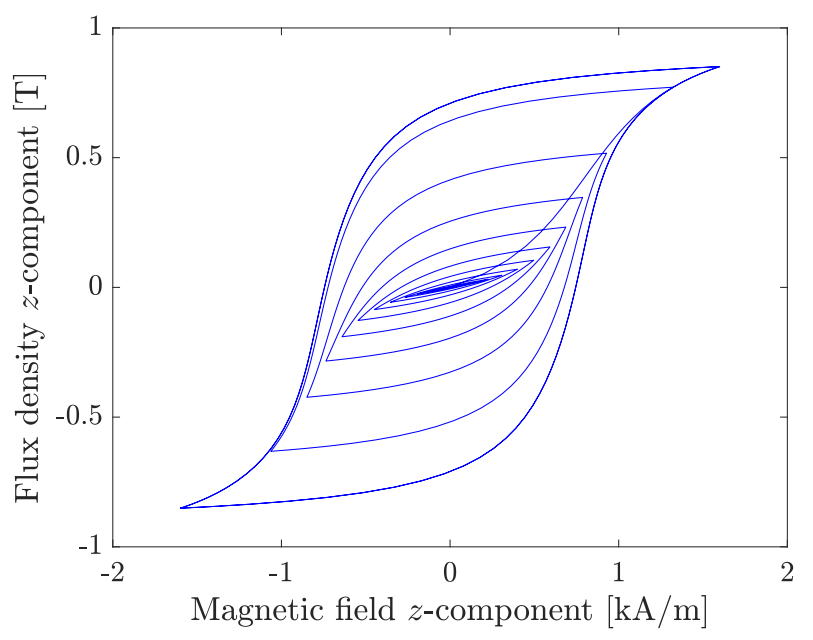

(b)

Fig. 17. Simulation IV-B2. (a) Time evolution of $B_{z}$ and $H_{z}$ at the center of the excited limb. (b) $B_{x}$ versus $H_{x}$ at the same point.

The properties chosen for the magnetic plate material are as follows.

1) $M_{\max }=1.4 \times 10^{6} \mathrm{Am}^{-1}$.

2) $H_{\text {ref }}=350 \mathrm{Am}^{-1}$.

3) $\Theta=1.2$.

4) $w_{1}=3$.

5) $w_{2}=0.45$.

The coil carries a sinusoidal current, denoted by $I_{\mathrm{S}}$ in Fig. 14, with a peak amplitude of $0.4 \mathrm{~A}$ and a frequency of $50 \mathrm{~Hz}$. The sampling frequency is $6 \mathrm{kHz}$. The equivalent circuit resulting from the chosen mesh consists of 48 nodes and 120 branches. The magnetic domain is discretized in 24 cells.

A simulation time of $0.16 \mathrm{~s}$ is considered. Fig. 15(a) shows the time evolution of the $B$ and $H x$-components recorded at the point $(x, y, z)=(5,5,0.45)[\mathrm{mm}]$. Fig. 15(b) shows the corresponding $B-H$ graph.

In this example, 960 time steps, involving 24 instances of the hysteresis model, are simulated in $308 \mathrm{~s}$, corresponding to an average of about $13.4 \mathrm{~ms}$ per time step per instance. 
2) Closed Magnetic Circuit: As a further example, a closed magnetic circuit is simulated. The system geometry is depicted in Fig. 16.

The current $I_{\mathrm{S}}$ is generated by an ideal source and flows through a coil with equivalent dc resistance of about $90 \mathrm{~m} \Omega$. The parameters chosen to characterize the hysteretic material of the core are listed as follows.
1) $M_{\max }=840 \times 10^{3} \mathrm{Am}^{-1}$.
2) $H_{\text {ref }}=800 \mathrm{Am}^{-1}$.
3) $\Theta=3$.
4) $w_{1}=3$.
5) $w_{2}=0.45$.

The sampling period is $\Delta_{\mathrm{t}}=0.2 \mathrm{~ms}$ and the discretized time variable is $t_{\mathrm{k}}=k \Delta_{\mathrm{t}}$, where $k=0,1, \ldots, 1500$. The electric current waveform is given by

$$
I_{\mathrm{S}}\left(t_{\mathrm{k}}\right)= \begin{cases}I_{0} \sin \left(2 \pi f t_{\mathrm{k}}\right), & t_{\mathrm{k}} \in\left[0, \frac{2}{f}\right) \\ I_{0} \sin \left(2 \pi f t_{\mathrm{k}}\right) e^{\frac{1}{\tau}\left(\frac{2}{f}-t_{\mathrm{k}}\right)}, & t_{\mathrm{k}} \in\left[\frac{2}{f}, \frac{15}{f}\right]\end{cases}
$$

where $I_{0}=500 \mathrm{~A}, f=50 \mathrm{~Hz}$, and $\tau=50 \mathrm{~ms}$. The analysis is performed by using a uniform mesh resulting in an equivalent circuit with 110 nodes and 408 branches. The number of magnetic volume cells is 192, meaning that 192 hysteresis model instances are involved in the simulation.

The time evolution of the $B$ and $H z$-components, probed at the center of the excited limb, is presented in Fig. 17(a), whereas Fig. 17(b) shows the corresponding $B-H$ curves. As expected, an inward spiraling $B-H$ path, commonly referred to as demagnetization curve, is obtained.

The elapsed real time for this example is about $2617 \mathrm{~s}$, corresponding to about $9.1 \mathrm{~ms}$ per time step per instance.

\section{CONCLUSION}

This paper has presented a new, fast, versatile, and robust inverse hysteresis model for circuit and electromagnetic simulations for a quasi-magnetostatic regime. The model has been developed on the basis of a direct model presented in a previous work. The need of an inverse version has arisen while investigating on the possibility to implement the hysteretic behavior of magnetic material into the PEEC framework in its formulation for nonlinear magnetic materials.

The proposed model is able to reproduce symmetric and asymmetric loops, initial magnetization curves, and reversal curves of any order. Its reliability and robustness have been demonstrated by testing it in stand-alone configuration and the Simulink environment. In the latter, the model has been adopted to simulate the hysteretic core of a custom inductor and transformer, proving the model reliability and low computational complexity. A comparison with measurement has also been provided.

The integration of the hysteresis model in the PEEC method has been achieved by exploiting the properties of the PEEC formalism for nonlinear isotropic magnetic material. Such a formalism has been made suitable to host the developed model by means of minor adjustments. Examples of simulations produced by the resulting formalism have been reported, hence demonstrating the versatility of the proposed hysteresis model.

\section{REFERENCES}

[1] G. Bertotti, Hysteresis in Magnetism. New York, NY, USA: Academic, 1998.

[2] E. D. Torre, Magnetic Hysteresis. Hoboken, NJ, USA: Wiley, 2000.

[3] I. D. Mayergoyz, Mathematical Models of Hysteresis. New York, NY, USA: Springer, 2011.

[4] J. Takács, Mathematics of Hysteretic Phenomena: The T(x) Model for the Description of Hysteresis. Hoboken, NJ, USA: Wiley, 2003.

[5] Y. Bernard, E. Mendes, L. Santandrea, and F. Bouillault, "Inverse Preisach model in finite elements modelling," Eur. Phys. J.-Appl. Phys., vol. 12, no. 2, pp. 117-121, 2000.

[6] D. C. Jiles and D. L. Atherton, "Theory of ferromagnetic hysteresis," J. Magn. Magn. Mater, vol. 61, nos. 1-2, pp. 48-60, Sep. 1986.

[7] J. V. Leite, A. Benabou, and N. Sadowski, "Accurate minor loops calculation with a modified Jiles-Atherton hysteresis model," COMPELInt. J. Comput. Math. Elect. Electron. Eng., vol. 28, no. 3, pp. 741-749, 2009.

[8] K. H. Carpenter, "A differential equation approach to minor loops in the Jiles-Atherton hysteresis model," IEEE Trans. Magn., vol. 27, no. 6, pp. 4404-4406, Nov. 1991.

[9] D. Miljavec and B. Zidarič, "Introducing a domain flexing function in the Jiles-Atherton hysteresis model," J. Magn. Magn. Mater, vol. 320 , no. 5, pp. 763-768, 2008.

[10] N. Sadowski, N. J. Batistela, J. P. A. Bastos, and M. Lajoie-Mazenc, "An inverse Jiles-Atherton model to take into account hysteresis in timestepping finite-element calculations," IEEE Trans. Magn., vol. 38, no. 2, pp. 797-800, Mar. 2002.

[11] S. E. Zirka, Y. I. Moroz, P. Marketos, and A. J. Moses, "Congruencybased hysteresis models for transient simulation," IEEE Trans. Magn. vol. 40, no. 2, pp. 390-399, Mar. 2004.

[12] S. E. Zirka and Y. I. Moroz, "Hysteresis modeling based on similarity," IEEE Trans. Magn., vol. 35, no. 4, pp. 2090-2096, Jul. 1999.

[13] S. E. Zirka, Y. I. Moroz, R. G. Harrison, and N. Chiesa, "Inverse hysteresis models for transient simulation," IEEE Trans. Power Del., vol. 29, no. 2, pp. 552-559, Apr. 2014.

[14] S. E. Zirka, Y. I. Moroz, N. Chiesa, R. G. Harrison, and H. K. Høidalen, "Implementation of inverse hysteresis model into EMTP-Part I: Static model," IEEE Trans. Power Del., vol. 30, no. 5, pp. 2224-2232, Oct. 2015.

[15] S. E. Zirka, Y. I. Moroz, N. Chiesa, R. G. Harrison, and H. K. Høidalen, "Implementation of inverse hysteresis model into EMTP-Part II: Dynamic model," IEEE Trans. Power Del., vol. 30, no. 5, pp. 2233-2241, Oct. 2015.

[16] A. Astorino, M. Swaminathan, and G. Antonini, "A new approach for magneto-static hysteresis behavioral modeling," IEEE Trans. Magn., vol. 52, no. 9, Sep. 2016, Art. no. 7301014.

[17] M. S. Pierce et al., "Disorder-induced magnetic memory: Experiments and theories," Phys. Rev. B, Condens. Matter, vol. 75, p. 144406, Apr. 2007.

[18] E. R. Madelung, "Über Magnetisierung durch schnellverlaufende Ströme und die Wirkungsweise der Rutherford-Marconischen Magnetdetektors," Ann. Phys., vol. 322, no. 10, pp. 861-890, 1905.

[19] R. G. Harrison, "Positive-feedback theory of hysteretic recoil loops in hard ferromagnetic materials," IEEE Trans. Magn., vol. 47, no. 1, pp. 175-191, Jan. 2011.

[20] A. Ruehli, G. Antonini, and L. Jiang, Circuit Oriented Electromagnetic Modeling Using the PEEC Techniques. Hoboken, NJ, USA: Wiley, 2017.

[21] D. Romano and G. Antonini, "Quasi-static partial element equivalent circuit models of linear magnetic materials," IEEE Trans. Magn., vol. 51, no. 7, Jul. 2015, Art. no. 7002115.

[22] D. Romano, G. Antonini, and A. E. Ruehli, "Time-domain partial element equivalent circuit solver including non-linear magnetic materials," IEEE Trans. Magn., vol. 52, no. 9, Sep. 2016, Art. no. 7004911. 